\title{
India's Clinical Trial Regulatory Changes, Indian Researcher's Awareness of Recently Changed Regulations, and the Impact of the New Drugs and Clinical Trial Rules: A Review
}

\author{
V. VENNU*AND P. P. SAINI ${ }^{1}$ \\ Department of Rehabilitation Sciences, College of Applied Medical Sciences, King Saud University, 10219 Riyadh, Saudi \\ Arabia, 1School of Pharmacy, Lingaya's Vidyapeeth, Faridabad-121002, India
}

Vennu et al.: Indian Clinical Trial Rules, Awareness, and Impact

\begin{abstract}
Medical companies consider India is above other countries worldwide as an alternative nation for clinical trials due to easily available sources and infrastructure. However, the developing Indian clinical trial industry has been negatively affected by noncompliance with regulations and by reports of unethical trials. Several studies on Indian clinical trial regulations have been conducted and various articles have been published, but only a few researchers evaluated awareness of investigators and members of the ethics committee about previously amended regulations. No study evaluated the knowledge of researchers on the new drugs and clinical trial rules issued in 2019 and also its impacts. Understanding the knowledge of Indian researchers on new drugs and clinical trial rules, including its effect is crucial to determine whether the trials are being conducted in compliance with the new rules and regulations. Thus, this review aimed to evaluate India's clinical trial regulatory changes based on the existing literature, Indian researcher's knowledge of the recent changes, and assessment of the impact of the new 2019 regulations, elaborating upon clinical trials in both the global and the Indian context.
\end{abstract}

Key words: Clinical trial, rules, regulation, researcher, India, drug, ethics committee, informed consent, and pharmaceutical

Because India is the second most populous developing country and encompasses various geographical conditions ${ }^{[1]}$, medical companies consider it above other countries worldwide as an alternative nation for clinical trials ${ }^{[2,3]}$. Principal justifications for performing clinical trials in India include a larger patient pool, a full spectrum of diseases, ethnically diverse populations, English-speaking health care professionals, low costs, and sound medical and information technology (IT) infrastructure ${ }^{[4,5]}$. In contrast, other developing countries pose various obstacles to clinical trials: commuting is difficult in Indonesia, communication is a significant problem in African countries, and in Western Europe, trials are expensive, patients have limited health issues, and language is a major barrier ${ }^{[6]}$. Thus, various multinational pharmaceutical companies have begun to consider outsourcing clinical trials to India ${ }^{[3]}$.

Currently, India is known as a hub for clinical trial companies, which consider the Indian market an

*Address for correspondence

E-mail: vvennu@ksu.edu.sa

September-October 2020 appropriate ground for clinical research ${ }^{[7,8]}$. However, the developing Indian clinical trial industry has been negatively affected by noncompliance with regulations and by reports of unethical trials ${ }^{[9-11]}$, even after several amendments to India's clinical trial rules ${ }^{[12]}$. Indian government authorities have recently addressed these issues by establishing regulative mechanisms for clinical trial review based on an in-depth investigation, media attention ${ }^{[13]}$, nongovernmental organization (NGO) involvement ${ }^{[10]}$, Supreme Court hearings, and recommendations from an expert committee ${ }^{[14]}$.

In 2019, to further advance clinical research in India and implement a routine, clear, and valid process for clinical trials $^{[6,15]}$, the New Drugs and Clinical Trial rules

This is an open access article distributed under the terms of the Creative Commons Attribution-NonCommercial-ShareAlike 3.0 License, which allows others to remix, tweak, and build upon the work non-commercially, as long as the author is credited and the new creations are licensed under the identical terms

Accepted 14 September 2020

Revised 02 July 2020

Received 18 February 2020

Indian J Pharm Sci 2020;82(5):726-740 
(NDCT Rules) were issued ${ }^{[16,17]}$. These rules reportedly aim to ensure the Indian population faster access to new drugs. Moreover, they increase the transparency of the regulative landscape for NDCT, which may generate quicker approvals.

Several studies on Indian clinical trial regulations have been conducted ${ }^{[18-22]}$ and various articles have been published ${ }^{[12,14,23-25]}$, but the evolution of opinions and perceptions among related professionals, such as research assistants, coordinators, associates, and managers, has been neglected ${ }^{[20,21]}$, as have these professionals awareness of and opinions on NDCT Rules.

Understanding the awareness and opinions of research professionals is crucial because they act as communicators and links between clinical trial sponsors and patients, making them instrumental in determining whether the trials are being conducted in compliance with the new drugs and clinical trial rules (NDTC Rules). Furthermore, they play an essential role in addressing, reporting, and documenting unethical clinical trials for investigators and ethics committees, particularly trials that involve vulnerable populations in India ${ }^{[26]}$. Therefore, this review aims to describe the changes in India's clinical trial regulations over the past decade, Indian researcher's awareness of the recently changed rules, and the impact of the newly issued 2019 regulations. This is done by elaborating upon clinical trials in both the global and the Indian context.

\section{CLINICAL TRIALS}

\section{Global context:}

According to Make et al. ${ }^{[27]}$, "Experimental discoveries are beginning with fundamental research, progress through pre-clinical animal studies, and then show therapeutic efficacy in human clinical trials". In other words, investigators require the most promising results from pre-clinical animal experiments examining a new molecular entity's preliminary efficacy, toxicity, and pharmacokinetics before approval is granted by a regulatory authority or ethics committee and clinical trials on human beings begin ${ }^{[28]}$. At that point, human trials determine whether the new drug is safe and effective for successful treatments. Importantly, regulatory authority approval does not mean that a therapy is safe or effective, only that a clinical trial is permitted. The regulatory body is responsible for vetting the risk/benefit ratio of the trial only ${ }^{[29]}$. Essentially, clinical trials are experiments or observations conducted in clinical research ${ }^{[4]}$. Prospective research studies involving human participants must provide specific information about biomedical or behavioral interventions, including the safety and efficacy of new treatments and interventions that warrant further research or comparison ${ }^{[4]}$.

Clinical trials vary in size and cost. They can involve a single research center or multiple, in one country or several. Clinical study design aims to ensure the scientific validity and reproducibility of the results. Costs can range into the billions of dollars per approved drug $^{[30]}$. Sponsors may be governmental organizations or pharmaceutical, biotechnology, or medical device companies. Certain functions vital to the trial, such as monitoring, oversight, or lab work ${ }^{[31]}$, may be managed by an outsourced partner such as a contract research organization (CRO) or a central laboratory. Of all drugs tested in human clinical trials, only $10 \%$ are ultimately approved by a national regulatory authority and marketed ${ }^{[27,32]}$.

\section{History:}

The concept of the clinical trial is ancient ${ }^{[33]}$, reaching as far back as biblical descriptions in the Book of Daniel in 500 BC of dietary therapy using legumes and lemons. After the basic clinical trial approach was described in the 18th century, continuing efforts were made to refine its design, statistical aspects, regulation, and ethical environment, with various major milestones in the evolutionary process ${ }^{[33]}$.

The history of clinical trials spans a wide variety of scientific, ethical, and regulatory challenges ${ }^{[33]}$. According to Dr. Arum Bhatt, who described the history of clinical research before and beyond James Lind $^{[33]}$, "the evolution of clinical research traverses a long and fascinating journey from the first recorded trial of legumes in biblical times to the first randomized controlled trial of streptomycin in 1946".

The first proper, systematic, controlled clinical trials were conducted in 1747 by the physician James Lind on the disease scurvy (vitamin C deficiency) ${ }^{[34]}$. Subsequently, the practice began to take its modern form $^{[35]}$. John Haygarth demonstrated the importance of a control group to correctly identify the placebo effect in his celebrated study of an ineffective remedy called Perkins Tractors $^{[36]}$. Further work in that direction was conducted in the 1860 s by the eminent physician Sir William Gul[ ${ }^{[36]}$. Frederick Akbar Mahomed, who was employed at Guy's Hospital in 
London, made substantial contributions to the clinical trial process when he distinguished chronic nephritis with secondary hypertension from what we now call essential hypertension ${ }^{[37]}$. He also founded the Collective Investigation Record for the British Medical Association, which collected data from physicians practicing outside hospital settings and served as a precursor to modern collaborative clinical trials.

Sir Ronald A. Fisher proposed the importance of randomization or the random assignment of individuals to different groups for an experiment ${ }^{[38]}$. In the 1930 s, the British Medical Research Council (MRC) established the Therapeutic Trials Committee to advise and assist in arranging properly controlled clinical trials ${ }^{[36]}$. In 1943, the MRC conducted the first double-blind controlled trial of patulin for the common $\operatorname{cold}^{[33]}$.

The first double-blind, placebo-controlled, randomized curative trial was conducted between 1946 and 1947 by Sir Geoffrey Marshall at the MRC Tuberculosis Research Unit ${ }^{[39]}$. The experiment aimed to test the effectiveness of streptomycin for curing pulmonary tuberculosis. This landmark trial was a model of meticulousness in design and implementation, with enrollment criteria and data collection processes that were highly systematic in comparison to the ad hoc nature of other contemporary research.

Sir Austin Bradford Hill, who was involved in the streptomycin trials, further developed the clinical trial methodology by applying statistics to medicine. He became famous for a landmark study in 1950, conducted in collaboration with Richard Doll that examined the correlation between smoking and lung cancer by comparing lung cancer patients with a matched control ${ }^{[40]}$. Throughout the $y$, as the practice of controlled trials grew in sophistication and influence, the streptomycin trial has been continually hailed as groundbreaking.

As Bhatt notes, several milestones can also be identified in the ethical advancement of clinical trials, namely the Nuremberg Code, the Declaration of Helsinki, the Belmont Report, and the 1996 International Conference on Harmonization (ICH) Good Clinical Practice (GCP) Guideline. Similarly, regulation of clinical trials began to increase as government authorities of the early 20th century began to recognize the need to control medical therapies. In the future, as scientific advancement continues, new ethical and regulatory challenges will continue to necessitate dynamic updates to the ethical and legal framework of clinical trials ${ }^{[33]}$.

\section{Types and phases:}

Investigators establish a research objective and classify clinical trials accordingly ${ }^{[41]}$. For example, in an observational study, the investigators observe subjects and measure their outcomes, but do not actively manage the study ${ }^{[42]}$. In an interventional study, the investigators apply an experimental drug, surgical procedure, medical device, diagnostic, or other intervention and compare the treated research subjects with those receiving no treatment or the standard treatment ${ }^{[42]}$ to assess how the subject's health changes.

Trials are classified according to their purpose $\mathrm{e}^{[41]}$. After human research approval is granted, the national regulatory authority organizes and monitors the trial's results according to type ${ }^{[41]}$. Table 1 describes the different types and purposes of clinical trials.

Clinical trials are typically conducted in four phases, with the exception of trials involving new drugs, which commonly include five ${ }^{[41]}$. Each phase involves a different number of subjects and has a different purpose, focusing on identifying specific effects. The drug approval process for each phase is treated as a separate clinical trial.

The process of new drugs through phases I to IV typically takes a decade or longer and often costs well over a billion dollars ${ }^{[43,44]}$. Typically, national regulatory authorities approve drugs that successfully pass through phases I to III for use in the general population ${ }^{[41]}$. The new drug's risks, benefits, or best uses are assessed after marketing in Phase IV trials. Table 2 summarizes the standard phases of clinical trials.

\section{Trial design:}

Clinical study design formulates trials for both experimental and observational studies in medical, clinical, epidemiological, and other types of research involving human subjects ${ }^{[49]}$. The usual objective of a clinical study is to establish the safety and efficacy of an intervention that is in development and not yet approved by a health authority ${ }^{[50]}$. However, interventions that have already been approved can also be involved in clinical trials to further investigate their long-term effects or cost-effectiveness. Fig. 1 presents an outline of clinical trial design types that have been defined and elaborated upon in several previous studies ${ }^{[51-53]}$.

Many factors must be considered when selecting a study design. Different types of studies are subject to different types of bias ${ }^{[54]}$. For example, recall bias 


\begin{tabular}{|c|c|c|}
\hline No. & Type & Purpose \\
\hline 1 & Prevention trials & $\begin{array}{l}\text { To prevent disease in people who have never had the disease, or to prevent the disease } \\
\text { from returning. }\end{array}$ \\
\hline 2 & Screening trials & To identify specific diseases/conditions. \\
\hline 3 & Diagnostic trials & To find better tests/procedures to diagnose a particular disease or condition. \\
\hline 4 & Treatment trials & $\begin{array}{l}\text { To test experimental drugs, new combinations of drugs, new approaches to surgery, or } \\
\text { radiation therapy. }\end{array}$ \\
\hline 5 & $\begin{array}{l}\text { Quality of life trials/ } \\
\text { supportive care trials }\end{array}$ & To improve the comfort and quality of care for people with a chronic illness. \\
\hline 6 & Genetic trials & To assess the prediction accuracy of genetic disorders. \\
\hline 7 & Epidemiological trials & To identify the general causes, patterns, or control of diseases in a large population. \\
\hline 8 & $\begin{array}{l}\text { Compassionate use trials/ } \\
\text { expanded access trials }\end{array}$ & $\begin{array}{l}\text { To provide partially tested or unapproved therapeutics to a small number of patients with } \\
\text { no other standard treatments. }\end{array}$ \\
\hline 9 & Fixed trials & $\begin{array}{l}\text { To study the outcome from } n \text { instances using existing data only during the trial's design. } \\
\text { The trial is not modified after it begins and results are assessed until the study is complete. }\end{array}$ \\
\hline 10 & Adaptive trials & $\begin{array}{l}\text { To quickly identify whether drugs have a therapeutic effect among patients for whom the } \\
\text { drug is appropriate. }\end{array}$ \\
\hline
\end{tabular}

TABLE 2: SUMMARY OF CLINICAL TRIAL PHASES

\begin{tabular}{|c|c|c|c|c|}
\hline Phase & Type & Focus & Primary goal & $\begin{array}{l}\text { Success } \\
\text { rate }\end{array}$ \\
\hline $0^{[45]}$ & $\begin{array}{l}\text { Human micro-dosing } \\
\text { trial }\end{array}$ & Pharmacokinetics & $\begin{array}{c}\text { To learn how a drug is processed in the body by giving a } \\
\text { minimal dose to } 10 \text { to } 15 \text { people }\end{array}$ & \\
\hline [[46] & $\begin{array}{l}\text { Human/clinical } \\
\text { pharmacology trial }\end{array}$ & Safety and dosage & $\begin{array}{c}\text { To identify the best dosage with the fewest side effects } \\
\text { by giving a low dose to a small group of } 20 \text { to } 100 \\
\text { healthy volunteers }\end{array}$ & $70 \%$ \\
\hline $\mathrm{la}^{[47]}$ & & Safety & $\begin{array}{l}\text { To confirm the safety of a single dose of the drug given } \\
\text { to small groups of subjects over time }\end{array}$ & \\
\hline $\mathrm{Ib}^{[47]}$ & & $\begin{array}{l}\text { Safety and } \\
\text { tolerability }\end{array}$ & $\begin{array}{l}\text { To investigate the pharmacokinetics and } \\
\text { pharmacodynamics of multiple doses of the drug in a } \\
\text { group of patients }\end{array}$ & \\
\hline $\begin{array}{l}\text { Food } \\
\text { effect }\end{array}$ & & Absorption & $\begin{array}{l}\text { To investigate differences in the body's absorption of } \\
\text { the drug caused by eating before the drug is given }\end{array}$ & \\
\hline$\|^{[48]}$ & Exploratory trial & $\begin{array}{l}\text { Efficacy and side } \\
\text { effects }\end{array}$ & $\begin{array}{c}\text { To evaluate biological activity or effects in larger } \\
\text { groups of } 100 \text { to } 300 \text { patients with specific diseases } \\
\text { once a dose or range of doses has been determined in } \\
\text { Phase I trials }\end{array}$ & $33 \%$ \\
\hline Ila & & Proof of concept & To demonstrate clinical efficacy or biological activity & \\
\hline IIb & & Dose-finding & $\begin{array}{l}\text { To find the optimal dose at which the drug shows } \\
\text { biological activity with minimal side effects }\end{array}$ & \\
\hline III[48] & Confirmatory trial & $\begin{array}{l}\text { Efficacy and adverse } \\
\text { reactions }\end{array}$ & $\begin{array}{l}\text { To assess the effectiveness and value of a drug in } \\
\text { clinical practice on large groups of } 300 \text { to } 3000 \text { or more } \\
\text { patients, depending upon disease/medical condition }\end{array}$ & $25-30 \%$ \\
\hline IV[41] & $\begin{array}{l}\text { Post-marketing } \\
\text { surveillance trial, } \\
\text { confirmatory trial }\end{array}$ & Safety and efficacy & $\begin{array}{c}\text { To test short-lived and long-lasting side effects and } \\
\text { monitor safety in several hundred or thousands of } \\
\text { patients after the drug receives national regulatory } \\
\text { authority approval }\end{array}$ & $\mathrm{N} / \mathrm{A}$ \\
\hline
\end{tabular}

is likely to occur in cross-sectional or case-control studies $^{[55]}$. In such studies, subjects are asked to recall exposure to risk factors. Furthermore, subjects with relevant conditions, such as breast cancer, may be more likely to remember the pertinent disclosures for a hormone replacement therapy they received than subjects without the condition. An ecological fallacy may occur when conclusions about individuals are drawn from analyses conducted on grouped data ${ }^{[56]}$, which tend to overestimate the degree of association between variables. Finally, seasonal variations and weather patterns can complicate seasonal studies on conditions such as allergies or influenza ${ }^{[57]}$. Scott R. Evans has previously summarized and discussed fundamental issues in clinical trial design in detail ${ }^{[50]}$. According to Scott, "most errors in clinical trials are a result of poorer planning, and the design flaws cannot rescue by fancy statistical methods. Thus, careful planning with clear foresight is crucial. Issues in trial conduct and analyses should be anticipated during trial design and thoughtfully addressed". 


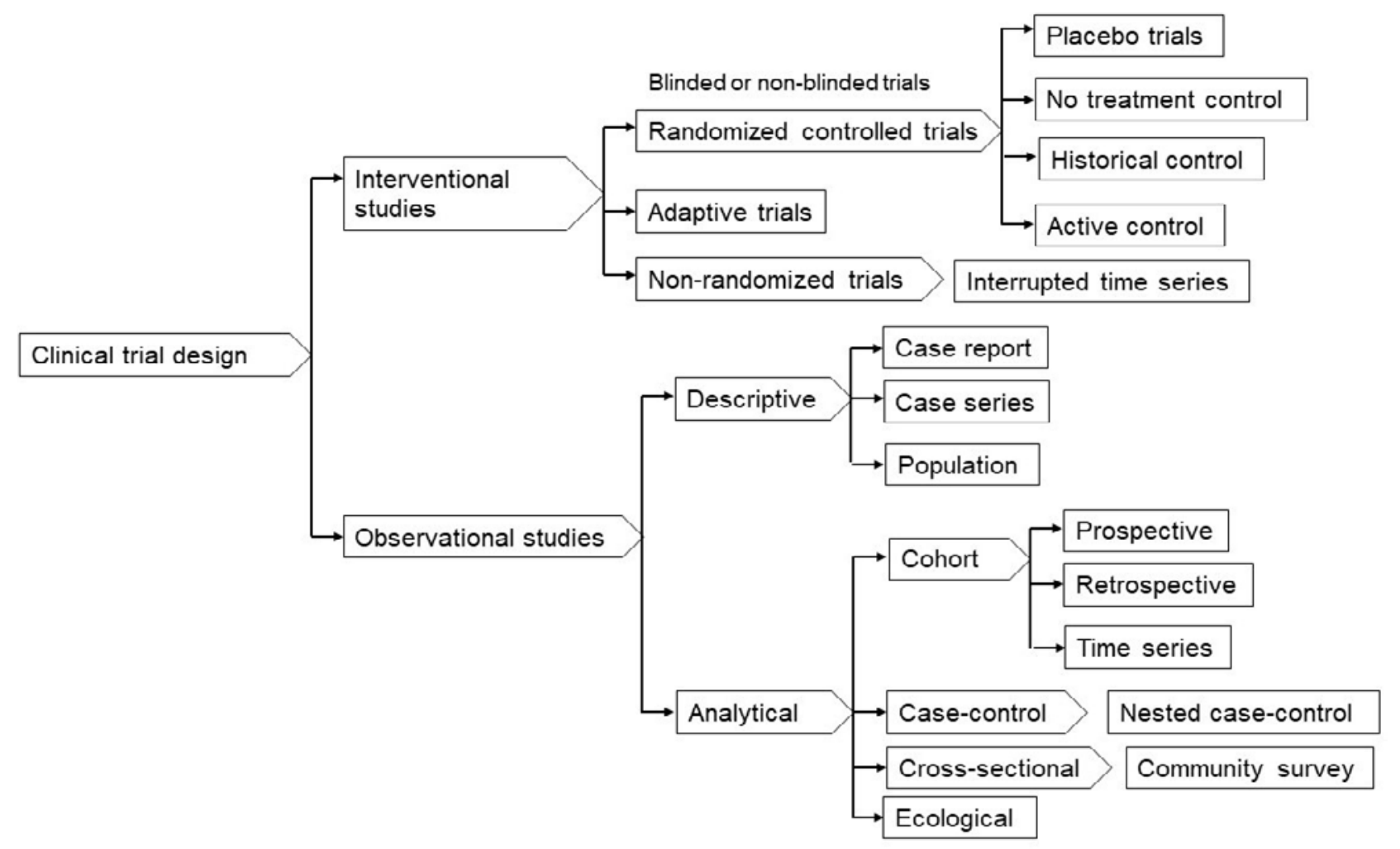

\section{Fig. 1: Flowchart outline of clinical trial design types}

Other trial design features include the protocol, informed consent, statistical power, and duration. The study protocol is a document prepared by a panel of experts to safeguard subjects' health and answer specific research questions ${ }^{[58]}$. It defines a precise, predefined procedural method for an experiment's design and implementation ${ }^{[58]}$ and specifies the detailed procedures, equipment, and instruments to be used; the study's objectives; the rationale behind the experimental design and chosen sample sizes; safety precautions and methods for calculating, interpreting, and reporting results ${ }^{[58,59]}$; statistical analysis; and any rules for predefining and documenting excluded data to avoid bias ${ }^{[58,59]}$. In other words, a protocol provides an exact template for the strict implementation of the trial by the investigators ${ }^{[58]}$. Globally, the United States, the European Union, and Japan have standardized protocol format and content to follow the GCP guidelines ${ }^{[60]}$ established by the ICH. Regulatory authorities in Canada and Australia also follow these guidelines.

In 1947, the Nuremberg Code set an international standard with 10 basic morals, ethical, and legal principles to prevent ethical violations ${ }^{[61,62]}$ (Lasker provides a detailed account of worldwide clinical research scandals and human experiment tragedies in the $19^{\text {th }}, 20^{\text {th }}$, and $21^{\text {st }}$ centuries, including the 10 basic principles of the Nuremberg Code, the Declaration of Helsinki, and the Belmont Report) ${ }^{[63]}$. The basic standards laid out for informed consent continued to develop and became a standard part of the research procedure $^{[64]}$. Informed consent is the process by which researchers respect the subjects' dignity and obtain permission before conducting a health care intervention or disclosing personal information. It is a core aspect of ethical clinical research ${ }^{[64]}$. After investigators explain all aspects of the trial that are relevant to subjects decision to participate, subjects voluntarily confirm their willingness to participate in that particular trial by providing a signature and date on an informed consent form ${ }^{[65]}$. However, although informed consent is a default practice in medical settings, it is not always required in research that involves low or no risk to participants ${ }^{[65]}$. Researchers consult with an ethics committee or institutional review board (IRB) to determine whether informed consent is needed for their trials ${ }^{[65]}$. For reference, informed consent form templates can be found on the World Health Organization (WHO) website ${ }^{[66]}$.

The number of subjects in a clinical trial, also known as sample size, has a major impact on the ability of the trial to reliably detect and measure the effects of the intervention ${ }^{[67]}$, known as the trial's "power". Power must be calculated before initiating an experiment to determine whether the cost is justified ${ }^{[68]}$. Broadly, statistical power estimates a trial's ability to detect a difference between the treatment and control 
groups of a particular sample size ${ }^{[67]}$. For example, a trial of a lipid-lowering drug versus a placebo with 100 patients in each group might have a power of 0.90 to detect a difference between the placebo and trial groups receiving a dosage of $10 \mathrm{mg} / \mathrm{dl}$ or more, but a power of only 0.70 to detect a difference when the trial group receives a dosage of $6 \mathrm{mg} / \mathrm{dl}$.

The duration of a clinical trial constitutes only a small part of the overall research involved in evaluating a drug for a new treatment. Potential drugs must be discovered, purified, characterized, and tested in labs (cell and animal studies) before even beginning clinical trials. Approximately 1000 potential drugs are tested before one reaches the clinical trial stage ${ }^{[69]}$. For example, new cancer drugs have an average of six y of research behind them before beginning clinical trials ${ }^{[70]}$. Nevertheless, the major obstacle to making new cancer drugs available is the time required to complete clinical trials. An average of eight y passes from the time a cancer drug enters clinical trials until it receives regulatory approval for sale to the public ${ }^{[70]}$. Drugs for other diseases have similar timelines.

The most significant barrier to completing studies is a shortage of potential participants ${ }^{[71]}$. It is challenging to find appropriate patients and obtain their consent to participate in a trial, especially when they may receive no direct benefit participants are not paid, the study drug is not yet proven to work, or the patient may receive a placebo in the trial. Although approximately 400 cancer drugs were tested through clinical trials in 2005 , approval was delayed because of the low numbers of participants ${ }^{[71]}$.

Usually, sponsor companies contract with CROs to perform all administrative work on a clinical trial. The CRO recruits participating researchers, such as assistants, coordinators, associates, and managers ${ }^{[72]}$, and trains them to coordinate study administration, collect data, set up meetings, monitor trial sites for compliance with the clinical protocol, and ensure the sponsor receives data from every site. These trained researchers do most of the work to conduct the clinical trial at a participating site ${ }^{[72]}$. They provide the local IRB with the documentation required to obtain study permission, assist with initiating the study, identify eligible patients and obtain consent from them or their families, administer study treatment(s), collect and statistically analyze data, maintain and update data files during follow-up, and communicate with the IRB, sponsor, and $\mathrm{CRO}^{[72]}$. Essentially, these research professionals act as communicators and links between the sponsors and patients of a clinical trial; their opinions are thus vital in determining whether trials are being conducted in compliance with the relevant regulations. Furthermore, their awareness of new directives is crucial because these professionals play an essential role in addressing, reporting, and documenting ethical violations in clinical trials for the investigators and ethics committee.

\section{Ethical aspects:}

The regulatory authorities of every nation closely supervise clinical trials to evaluate the integrity of their data, protect patient's safety, and assess the adequacy of sites and sponsors ${ }^{[73]}$. A supervising IRB or ethics committee must grant advance approval for studies involving a medical or therapeutic intervention for patients, especially when the research concerns vulnerable populations ${ }^{[62]}$. One of the IRB's primary functions is to ensure potential patients are adequately informed about the clinical trial[ ${ }^{[62]}$. Therefore, to satisfy ethical standards, researchers must obtain informed consent from the research participants. If patients are unable to provide consent for themselves, researchers can seek approval from their legally authorized representatives. Borovecki discusses the history, types, functions, structure, and locale of research ethics committees, along with the global perspective of their $\operatorname{work}^{[74]}$.

In response to conflicts of interest, the Pharmaceutical Research and Manufacturers of America published new guidelines urging companies to report all findings and limit the financial involvement in drug companies by researchers ${ }^{[75]}$. Although pharmaceutical companies do not directly employ drug researchers, they often seek grants from manufacturers. These manufacturers, in turn, often look to academic researchers to conduct studies within their university and hospital networks. Similarly, competition for tenured academic positions, government grants, and prestige creates conflicts of interest among academic scientists ${ }^{[76]}$. However, approximately $75 \%$ of articles retracted for misconductrelated reasons have declared no industry financial support $^{[77]}$.

Aggregating safety data across clinical trials during drug development is important because trials are generally designed to determine how well a drug works ${ }^{[78]}$. Based on an aggregate safety assessment, decisions can be made throughout the drug's developmental process ${ }^{[78]}$. Sponsors and regulators can also better assess the drug's safety after it is approved. 
Despite the potential disadvantage of lower-quality trial management, many American pharmaceutical companies have moved clinical trials overseas ${ }^{[79]}$. The benefits are lower costs in some overseas countries and the ability to run more extensive trials in shorter timeframes. An estimated $40 \%$ of all clinical trials now take place in Eastern Europe, Central and South America, and Asia, including India ${ }^{[80]}$. However, according to Jacob Sijtsma of the Netherlands-based Wemos Foundation, a health advocacy organization that tracks clinical trials in developing countries, "There is no compulsory registration system for clinical trials in these countries and many do not follow European directives in their operations" ${ }^{[80]}$.

\section{Indian context:}

India has a long history in the field of clinical research and the rich heritage of traditional medicine, such as Ayurveda $^{[33]}$. The classical Ayurveda texts contain detailed observations on diseases and in-depth guidance on remedies. These descriptions are likely based on direct comments made by ancient Ayurveda experts. The ancient texts do not contain any recorded documentation of clinical experiments, but other major historical milestones of medical research in India have been identified ${ }^{[33,81]}$. An article on clinical research methodology by Purvi Gandhi also provides an informative review of the literature on clinical research methods including ethics, phases, design, and statistical aspects, such as p-value, significance level, error types, study power, sample size, and data analysis ${ }^{[54]}$.

Recently, clinical research has become globalized ${ }^{[82]}$. Consequently, many clinical trials are conducted in developing countries like India ${ }^{[33]}$ to overcome barriers faced in countries like the United States, where studies may fail to enroll the desired number of participants due to strict regulations, extensive safety requirements, compensation obligations, and extremely high $\operatorname{costs}^{[4]}$. Recently, India has gained recognition as an attractive country for clinical trials and a highly promising research hub ${ }^{[33,83]}$. Many critical success factors for expanding clinical research are present in $\operatorname{India}^{[4]}$. However, lack of GCP training among stakeholders, the weak or favorable regulatory system, and unethical human experimentation have driven many revisions to India's rules and regulations for NDTC ${ }^{[4,84,85]}$.

\section{HISTORY OF INDIA'S CLINICAL TRIAL REGULATIONS}

In addition to the specific critical success factors that attract global stockholders to expand clinical research in
India, over the past decade, a robust regulatory system has been developed that places India in compliance with global regulatory guidelines. In a previous article, Hitt Sharma and Sameer Parekh illustrate a flowchart of India's regulatory bodies and outline their roles in standardizing clinical research in India by regulating drug manufacturing, sales, clinical trials, imports, and exports ${ }^{[86]}$. The history of drug regulation, in particular, goes back to the British colonial era.

In 1940, the Indian government formed a drug inquiry pane $^{[25]}$. Its recommendations were later presented for discussion in the legislative assembly as the Drug Bill, which became the Drugs and Cosmetic Acts of $1940^{[87]}$ and, later, the Drugs and Cosmetics Rules of 1945. These fundamental acts still regulate imports, manufacturing, distribution, and sale of drugs and cosmetics in India. The Drug Controller General of India (DCGI), under the division of the Ministry of Health and Family Welfare, grants permission to conduct human clinical trials of new drugs in the country.

The Indian Patent Act of 1970 made provisions in India only for "process" patents ${ }^{[88]}$. However, in 1994, the Indian government signed the affidavit on Trade-Related Aspects of Intellectual Property Rights (TRIPS), which protects the intellectual property (IP) rights of the member states of the World Trade Organization (WTO) ${ }^{[25]}$. In 2005, to attract multinational pharmaceutical companies, India amended its Patent Bill for pharmaceutical products and broadened its process to strengthen the TRIPS competent "product" patent system ${ }^{[25]}$. Consequently, western companies have no concern about marketing new drugs to Indian markets; the revisions to India's Patent Bill prohibit Indian companies from duplicating patented drugs by making small changes to the production process.

Recognizing the benefits of clinical research for new therapies, India's government has developed various ethical and regulatory guidelines through a regulatory agency known as the Indian Council of Medical Research (ICMR) ${ }^{[89]}$. The ICMR formulates, coordinates, and promotes biomedical research in India. The 2000 and 2006 ICMR guidelines ${ }^{[90]}$ for biomedical research on human subjects mandate ethics committees at the institutional level and require researchers to obtain ethical approval from an appropriate committee before submitting a study proposal to the DCGI. However, the adoption of an ethics committee is not mandatory to conduct a clinical trial in India according to the 2006 guidelines. 
The Central Drugs Standard Control Organization (CDSCO) released the Indian GCP guidelines in 200 ${ }^{[25]}$. The guidelines initially mandated that a Phase II trial could be conducted only if the Phase III study was proceeding elsewhere ${ }^{[85]}$. This step was taken to ensure that drug safety data were available and that Indians were not the only recipients of experimental drugs. However, most pharmaceutical organizations did not follow the GCP guidelines at that time, resulting in low quality data and phase lag, which harmed the country's reputation in the field. Therefore, in 2005 the CDSCO amended Schedule Y of the Drugs and Cosmetics Rules of $1945^{[25]}$ to eliminate phase lag and provide a collection of clinical trial guidelines and regulations. Details of these modifications have been described previously ${ }^{[25,91]}$. Furthermore, in 2006, the CDSCO issued a fast-tracking system to decrease the application review period from 16 to $10 \mathrm{w}$, separating clinical trial applications into class $\mathrm{A}$ or $\mathrm{B}^{[22]}$. Class $\mathrm{A}$ applications, almost all Phase III trials, have received approval from competent and fully-fledged regulatory systems in countries such as the United States, Japan, Canada, European countries, Australia, and Switzerland; these qualify for fast-track review in India, which takes two to four $\mathrm{w}$ or fewer. All other applications fall under class B, for which approval takes approximately $12 \mathrm{w}$.

In 2007, India's government eliminated the $12 \%$ service tax on clinical trials ${ }^{[25]}$ and established a free, online portal for clinical trial registration, the Clinical Trials Registry of India (CTRI) ${ }^{[93]}$. Registration was not mandatory at that time but was made compulsory as of June 2009 ${ }^{[93,94]}$. The CTRI portal allows registration of both investigator-initiated and regulatory studies to be registered ${ }^{[93]}$; it is recommended that all studies be registered in CTRI's public portal ${ }^{[93]}$.

Despite the numerous amendments to India's clinical trial regulations between 2005 and 2015 $5^{[93]}$, many loopholes remained ${ }^{[24]}$. India's failure to implement its ethical guidelines was observed in many human rights violations committed by researchers ${ }^{[25]}$. Additionally, instances of scientific misconduct, such as falsification of informed consent, experimental data, or personal qualifications, were commonly reported ${ }^{[57,81]}$, even in the popular press $^{[80]}$.

\section{UNETHICAL HUMAN EXPERIMENTATION IN INDIA}

India is an emerging hub for clinical trials worldwide, with an estimated clinical trial market of over 20 billion Indian rupees, growing at $30 \%$ per $\mathrm{y}^{[4]}$. Furthermore, the average cost of a clinical trial in India (100 million US dollars) is considerably lower than in the United States (180 million US dollars) ${ }^{[95]}$. In the field of clinical research on humans, the pharmaceutical industry is one of the most critical players. "Human experimentation is experimentation conducted on human beings to test an idea or hypothesis. Researchers must compulsorily have their proposal approved by a relevant Ethics Committee"[96].

Incomplete and biased unethical trials on human beings have been conducted around the world ${ }^{[61]}$, in both developed and developing countries ${ }^{[4,97]}$. In some cases, the trials were not approved by an ethical review committee or IRB, or approval was granted despite an unethical trial design ${ }^{[4]}$. The research organizations involved have ranged from relatively unknown local companies to leading multinational corporations a surprising fact, given that multinational corporations usually have clear public commitments to uphold high ethical standards in clinical trials.

It is sometimes argued that unethical clinical trial cases are isolated or dated, but this is not always the case. Some unethical experiments have been conducted recently ${ }^{[80]}$; some are still being conducted ${ }^{[4]}$. Additionally, some older cases have been included in this overview because developments following them are ongoing. Finally, the nature of ethical concerns is diverse and relates to all paragraphs of the Declaration of Helsinki, and the ICMR GCP guidelines on human research $^{[98]}$. The most common issues and challenges regarding unethical human experimentation in India have been described previously ${ }^{[4]}$.

\section{History:}

In recent $y$, the number of clinical trials conducted in India has increased dramatically ${ }^{[86]}$. Clinical trials approved by the CDSCO between 2010 and 2014 have been reported previously ${ }^{[24]}$. But are all the human clinical trials undertaken in India conducted ethically? On the contrary, a large number of experiments were found to have been conducted on Indian patients without following the mandatory legal and ethical principles ${ }^{[99]}$. This may be because most Indians do not have access to good quality, affordable care, and might, therefore, accept offers to provide free and better quality treatment ${ }^{[100]}$. Thus, unethical experiments were conducted on people who were vulnerable because they could not afford proper quality treatment or the most effective drugs ${ }^{[101]}$. Patients were also vulnerable because they were seriously ill. Psychiatric patients ${ }^{[102]}$, 
for example, may not have been able to provide informed consent for clinical trials. Some of the most alarming examples of unethical trials, however, were those using experimental drugs that had not yet been established as safe for testing in humans. A few specific examples of unethical human experimentation in India are summarized below.

In the 1930s and 1940s, the British Army used hundreds of British and Indian soldiers in experiments to determine whether mustard gas inflicted more significant damage to Indian or British $\operatorname{skin}^{[103]}$. It is unclear whether the trial subjects, some of whom were hospitalized by their injuries, were all volunteers. In 1984, the Bhopal gas leak incident, considered one of the world's worst industrial disasters, occurred in India ${ }^{[104]}$. Victims of the disaster have been subjected to unauthorized clinical trials without their consent to benefit various pharmaceutical companies.

From 1994 to 1996, "hundreds of doctors" were involved in Quinacrine sterilization in India. In 1998, a trial conducted in West Bengal to test the anti-malarial drug Quinacrine as a contraceptive for women stirred up a furor ${ }^{[105]}$. A sustained campaign by women activists claimed that over 30000 women were sterilized with Quinacrine over five y, without permission from the health authorities. In a parliamentary session, the minister of state in the Department of Legal Affairs stated that the Indian Government was aware of the WHO's explicit recommendation to immediately cease trials of Quinacrine on human populations pending further study. He further stated that the government had granted permission for the ICMR to conduct a study of the drug in 1992, which was terminated due to the longterm effects and high failure rate early in the study, and subsequently, "approval for clinical trials of Quinacrine pellets had not been granted to any investigator by the DCGI".

Around 1999, Otsuka sponsored trials of Cilostazol for treatment of intermittent claudicating, which were cleared by the DCGI based on incomplete, inadequate information that did not report serious adverse events ${ }^{[106]}$. In 2000, Solvay Pharmaceuticals conducted trials of Cilansetron, a proprietary new molecule, for treatment of diarrhea due to irritable bowel syndrome. A Phase III trial was cleared by the DCGI even though only Phase II trials had been conducted abroad, and the drug was not approved anywhere in the world. At that time, Schedule $\mathrm{Y}$ of the Indian Drugs and Cosmetics Rules prohibited clinical trials of drugs developed outside India before completion of Phase II trials abroad. Ultimately, in April
2005, the FDA issued a non-approval letter regarding Solvay's marketing application for Cilansetron and requested additional clinical trials. In November 2005, Solvay withdrew its New Drug Application (NDA) in the United States. The company also filed a marketing application for Europe, which is still undergoing review in the United Kingdom ${ }^{[107]}$. A similar example is Pfizer's trials of Zoniporide for the treatment of preoperative cardiac events. The DCGI approved a Phase III trial of Zoniporide even though Phase II trials had not been completed in the United States and animal studies of the drug's carcinogenic and reproductive effects, which were mandated by Indian law, had not been completed ${ }^{[10]}$.

In 2002, Novo Nordisk conducted trials of Ragaglitazar for the treatment of diabetes in 32 countries, including India. The trials were suspended after urinary bladder tumors were discovered in a mouse and several rats treated with the drug. In India, 130 people from eight centers had participated in the trials, half of whom received the experimental drug. There was some dispute on whether the required animal experiments had been completed. Under ICMR regulations, the results of toxicity studies of medicines for chronic diseases were required before beginning Phase III clinical trials; this was not required in the EU and the US. Ultimately, Novo Nordisk conducted a clinical follow-up program that indicated no relation between Ragaglitazar exposure and cancer in the trial participants ${ }^{[106]}$.

In Gujarat, the healthy 22 y old "volunteer" Dharmesh Vasava was among many daily-wage workers who died while participating in tests of Citalopram, an antipsychotic drug sponsored by Mumbai-based Sun Pharmaceuticals ${ }^{[10]}$. Dharmesh developed pneumonia and died during the trial. According to another participant, subjects were induced to participate with money by agents of Sun Pharmaceuticals.

In 2003, Sun Pharmaceuticals conducted trials of Letrozole, during which over 400 women who had been trying unsuccessfully to conceive were enrolled without their knowledge or consent to participate in a clinical trial conducted at nine or more centers across India ${ }^{[10]}$. Subjects were not informed they were participating in a trial and did not provide informed consent. A complaint about the Letrozole case was filed with the Supreme Court by Social Jurist, a Delhi-based NGO.

In the case of streptokinase trials, which tested a "clot-busting drug" for use in heart attacks or diabetes, Shanta Biotechnics (streptokinase) and Biocon (insulin) openly conducted illegal Phase III clinical trials on 
unaware patients and failed to obtain informed consent or permission from the Genetic Engineering Approval Committee (GEAC) ${ }^{[108]}$. Neither was the trial protocol reviewed by an ethical review committee. Eight patients died during the trials, though Shanta Biotechnics denied the allegations. Litigation was filed by the Delhi-based NGO Aadar Destitute and Old People's Home. In March 2004, the Supreme Court of India confirmed that the trials were illegal.

Johnson \& Johnson's Risperidone trials in Gujarat for the treatment of acute mania were controversial because patients who received a placebo could suffer unnecessary harm from being taken off their standard medication $^{[109]}$. Furthermore, not all subjects were informed of their participation; one patient explained that he signed a form because the doctor wanted him to, but had no idea he was participating in a clinical trial. Thus, informed consent was not properly obtained from all participants, the use of a placebo was unnecessarily dangerous, not all patients received an explanation that the provided medical care was linked to research. Johnson \& Johnson denied these allegations and stated that consent was obtained from every patient. It defended the placebo-controlled trial design, claiming that it exposes fewer patients to potentially ineffective treatments. However, this did not justify patients having to discontinue a proven existing treatment.

In 2008, an infant in Bangalore with a pre-existing cardiac condition died while participating in a pneumococcal vaccine trial meant to recruit only healthy infants (Pandeya). In Hyderabad, a young adult who died in a bioequivalence trial of a blood pressure drug had reportedly participated in several bioequivalence trials (Ramana). The human papillomavirus (HPV) vaccine trial funded by the Gates Foundation was also revealed to have conducted gross ethical violations ${ }^{[110]}$. The trial, suspended in March 2010, was conducted by the Program for Appropriate Technology and Health (PATH), an NGO, in collaboration with the ICMR and the governments of Andhra Pradesh and Gujarat. It included nearly 23500 girls between 9 and 15 y of age in the Khammam district (Andhra Pradesh) and Vadodara (Gujarat). The "casual approach" of the trial led to the informed consent form being filled "very carelessly' with 'incomplete and probably inaccurate' information. In Andhra Pradesh, nearly 2800 informed consent forms were signed by a hostel warden or headmaster as the subject's "guardian."

Although the Indian market is an appropriate ground for clinical research ${ }^{[7,8]}$, the developing Indian clinical trial industry was affected by noncompliance with regulations and specific reports of unethical clinical trials like those described above $\mathrm{e}^{[9-11]}$. Thus, Indian government authorities have recently sought to address these issues by establishing regulative clinical trial review mechanisms based on intensive investigation, media attention ${ }^{[13]}$, NGO involvement ${ }^{[10]}$, and Supreme Court hearings ${ }^{[14]}$.

\section{RECENT DRUG AND CLINICAL TRIAL REGULATORY CHANGES IN INDIA}

In 2013, clinical research in India experienced dramatic change with the Ministry of Health and Family Welfare's introduction of a series of stringent guidelines and laws ${ }^{[111]}$. In other words, India has taken steps to guard against potentially dangerous or unethical clinical trials and to prevent the enrollment of patients without their consent by strengthening the regulatory mechanisms for clinical trial review. The new regulations also ensure that participants who experience adverse events are compensated ${ }^{[14]}$. All regulatory changes have been outlined ${ }^{[93]}$ and discussed previously, particularly the changes made in $2013^{[112]}$.

Despite the dramatic changes made in $2013^{[111]}$, clinical research in India has come under intense global scrutiny, with concerns being raised regarding the regulatory and ethical oversight of clinical trials conducted in the country ${ }^{[13]}$. Furthermore, a Public Interest Litigation was filed by an NGO alleging unethical clinical trials conducted in India on children, women, and mentally disabled patients ${ }^{[114]}$. In response, an expert committee was formed under Professor Ranjit Roy Chaudhary to review India's existing policies and guidelines for approval of new drugs, banning of drugs, and clinical trials $^{[115]}$. In 2016, to restore faith in the Indian clinical trial industry, the Ministry of Health and Family Welfare issued three consecutive amendments to the Drugs and Cosmetics Rules based on the recommendations given by the committee and directives issued by the apex court ${ }^{[116]}$. These amendments have been outlined in previous articles ${ }^{[20,93]}$.

On March 19, 2019, a draft of the NDCT Rules was published by the central government in the Gazette of India under the aegis of the Drugs and Cosmetics Act of $1940^{[117]}$. These rules aim to advance clinical research in India by implementing a standard, clear, and valid process for clinical trials ${ }^{[16,17]}$. Salient features have been summarized elsewhere ${ }^{[118]}$. The NDCT Rules are essential for uninterrupted and transparent implementation of clinical studies in India, which may 
lead to quicker approvals. Recently, papers have sought to summarize the crucial changes in the new rules and to assess the rules from an ethical perspective $\mathrm{e}^{[119]}$.

\section{Researcher's awareness of and opinions on recent regulatory changes:}

The 2013 guidelines posed a significant challenge to India's clinical trial industry ${ }^{[111]}$. Concerning the regulatory guidelines issued in 2016, a previous study has already assessed Indian investigators perceptions and opinions ${ }^{[20]}$. Responses to that web-based survey demonstrated general acceptance, belief ineffectiveness, and anticipated compliance with new regulatory guidelines among Indian researchers. About $90 \%$ of the respondents endorsed the guidelines on the registration of institutional and independent ethics committees; $76.8 \%$ endorsed guidelines on compensation for studyrelated standard adverse events; $49.3 \%$ endorsed restriction of the number of trials per investigator. The issue of audio-video (AV) consent was debated ardently. Although around $36 \%$ of investigators supported AV recording and $12 \%$ were neutral, approximately $50 \%$ were opposed. The majority of investigators $(49.3 \%)$ were undecided regarding the obligation to include government sites in clinical trials.

The Ethics Council of the Indian Society for Clinical Research also conducted a questionnaire survey of ethics committee members to examine the impact of the 2016 regulatory changes on the functioning of ethics committees, assessing members' awareness of the recent changes and effects and their opinions on the guidelines regarding serious adverse events, compensation, informed consent, AV recording, monitoring, auditing, and future ethics committee work $^{[21]}$. The results indicated that the regulatory changes empowered ethics committees in some ways, but also introduced challenges to providing oversight and monitoring research conducted at a trial site. The majority of members opposed the concept of a centralized ethics committee for review, approval, and oversight of clinical studies.

Recently, the present author conducted a web-based survey of Indian researcher's awareness of and opinions on the new regulations issued in 2019. In this crosssectional online survey, Indian researchers (men and women) were randomly selected from the four national zones (East, West, North, and South) and multiple sources between July and September 2019. The various sources included the Indian Society for Clinical Research, personal networks, hospitals, institutions, and LinkedIn. Participants were defined as researchers, co-researchers, assistants, coordinators, associates, ethics board members (chairperson, secretary, or other), and managers.

The results of the survey were as follows. Of the 106 total participants, 75 researchers $(70.8 \%)$ and 65 managers and clinical staff $(61.3 \%)$ understood the new regulations. A total of 36 researchers $(63.2 \%)$ supported a reduction in timelines, $32(53.1 \%)$ supported free post-trial drug access, and $31(54.5 \%)$ supported greater equality. New changes to the handling of severe adverse effects and compensation were supported by 37 researchers (64.9\%). Overall, 34 researchers $(59.6 \%)$ agreed with the new rules, $71(67 \%)$ felt that the new regulations would primarily impact for-profit clinical trial studies, and $91(87.5 \%)$ considered the new rules are highly favorable to promote clinical research in India.

\section{Impact of new regulatory amendments:}

India is a significant ground for clinical trials. While trials are in progress in India, regulations must ensure the safety and wellbeing of the study's subjects. A previous study has highlighted the effects of the amended rules on clinical trials registered for DCGI approval ${ }^{[21]}$. This study reports that a total of 1799 trials were approved, with a median rate of 206 per y, between 2007 and 2014. It also illustrated trends in clinical trials approved in India over the same period.

In contrast, a recent search of the CTRI shows a total of 7937 trials approved between 2007 and February 5, 2020. Trends in trials approved in India between 2007 and 2019 are illustrated in fig. 2. Due to the amendments made to clinical trial regulations, clinical trials in India increased substantially over the 13 y following 2007, and particularly after 2016 . Thus, the new rules issued in March 2019 have the potential to improve ethical standards, speed up clinical trials, and hasten the approval of new drugs ${ }^{[15]}$. This will help to expand clinical research and the pharmaceutical industry in India even more substantially.

The new regulatory changes of 2019 have additional impacts, particularly on ethics committees involved with clinical trials. To comply with the new rules, several ethics committees may have to be reconstituted. The increased period of validity granted to ethics committees will allow them to function for a longer duration if a renewal application is submitted on time. Additionally, the increased registration period may simplify the cumbersome process of registration renewal 


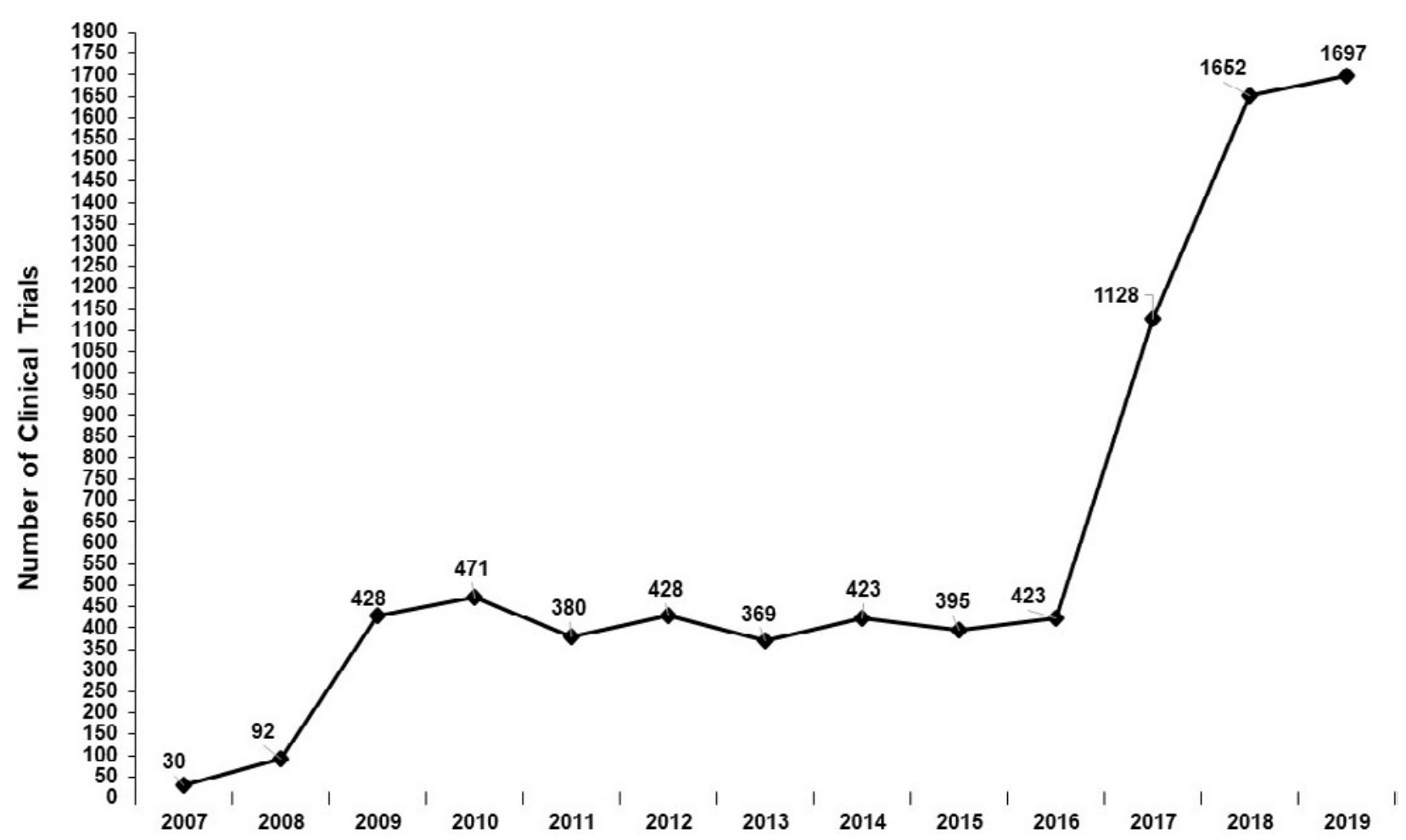

Fig. 2: Trends in clinical trials approved by the Drug Controller General of India from 2007 to 2019

for ethics committees, promoting greater compliance. Academic institutions should reconstitute existing ethics committees to create separate committees for clinical trials and academic research.

The new rules specify timelines for review of the newly mandated applications. However, per an order issued by the CDSCO on April 10, 2019, requests can be manually completed and uploaded to the SUGAM online submission portal until all the new forms are integrated into the platform. The new rules also specify a process for appeals and reconsideration of rejected applications. In general, clinical trials must be initiated within two y of acquiring permission. Finally, the new rules provide a more practical procedure for compensation in case of injury or death during a clinical trial by changing the wording from "the day of occurrence of a serious adverse event" to "knowledge of the occurrence of a serious adverse event".

This review aimed to evaluate India's clinical trial regulatory changes based on the existing literature, Indian researcher's knowledge of the recent changes, and assessment of the impact of the new 2019 regulations, elaborating upon clinical trials in both the global and the Indian context. Overall, this review's evaluation of the literature and examination of Indian researcher's knowledge highlights the importance of the NDCT Rules. Additionally, this review provides a more detailed, literature-based insight into India's clinical trial regulatory changes, including the most recently changed rules. This evolution will be of interest to stakeholders seeking to expand India's clinical trial and pharmaceutical industries. However, due to a lack of relevant literature, this review could not evaluate Indian researcher's knowledge of rule changes between 2007 and 2013. Despite this limitation, this study concretely adds to our understanding of India's recently amended regulations and the impact of the NDCT Rules. This topic is also a fruitful area for further work to fully understand the implications of the NDCT Rules.

\section{Acknowledgments:}

The authors extend their appreciation for support to the Research Center, College of Applied Medical Sciences and also the Deanship of Scientific Research and Researchers Support Services Unit at King Saud University for their technical support.

\section{Conflict of interests:}

The authors declared no conflict of interests.

\section{REFERENCES}

1. Rick Gladstone. India will be the most populous country sooner than thought, U.N. Says. New York Times; 2015.

2. Bajpai V. Rise of clinical trials industry in India: An analysis. Int Sch Res Notices 2013.

3. Mondal S, Abrol D. Clinical trials industry in India: Asystematic review. Institute for Studies in Industrial Development; 2015. 
4. Maiti R, Raghavendra M. Clinical trials in India. Pharmacol Res 2007;56(1):1-10.

5. Shenoi A. Biomedical research in India: law and ethics at crossroads. Soc Change 2012;42(4):527-38.

6. Drugs and Clinical Trials Rules, 2019. Drishtiias.com; 2019.

7. Fernandes SD, Anoop NV, Castelino LJ, Charyulu RN. A national approach to pharmacovigilance: The case of India as a growing hub of global clinical trials. Res Social Adm Pharm 2019;15(1):109-13.

8. Porter G. Regulating clinical trials in India: the economics of ethics. Dev World Bioeth 2018;18(4):365-74.

9. Network SA. Clinical trials in India: ethical concerns. Bull World Health Organ 2008;86(8):581-2.

10. Gulhati CM. Needed: closer scrutiny of clinical trials. Indian J Med Ethics 2004;24;1(1):4-5.

11. Yee A. Regulation failing to keep up with India's trials boom. Lancet 2012;379(9814):397-8.

12. Lahiry S, Sinha R, Choudhury S, Mukherjee A, Chatterjee S. Paradigm shift in clinical trial regulations in India. Indian $\mathrm{J}$ Rheumatol 2018;13(1):51-5.

13. Ramamurthy NV. Inept media trials of clinical trials. Perspect Clin Res 2012;3(2):47.

14. Chatterjee $P$. India tightens regulation of clinical trials to safeguard participants. Br Med J 2013;346:f1275.

15. Vaidyanathan G. India's clinical-trial rules to speed up drug approvals. Nature; 2019.

16. Jesani A, Srinivasan S. New Drugs and Clinical Trials Rules, 2019: The market trumps ethics and participant rights. Indian J Med Ethics 2019;4(2);89-91.

17. India: The new drugs and clinical trial rules, 2019. Mondaq Ltd; 2019.

18. Ramalingam S, Bhuvaneswari S, SanKaRan R. Ethics workshops-are they effective in improving the competencies of faculty and postgraduates? J Clin Diagn Res 2014;8(7):XC01.

19. Burt T, Sharma P, Dhillon S, Manchanda M, Mittal S, Trehan N. Clinical research environment in India: Challenges and proposed solutions. J Clin Res Bioeth 2014;5(6):1-8.

20. Kadam R, Borde S, Madas S, Nagarkar A, Salvi S, Limaye $\mathrm{S}$. Opinions and perceptions regarding the impact of new regulatory guidelines: A survey in Indian Clinical Trial Investigators. Perspect Clin Res 2016;7(2):81-7.

21. Davis S, Sule P, Bughediwala M, Pandya V, Sinha S. Ethics committees and the changed clinical research environment in India in 2016: A perspective. Perspect Clin Res 2017;8(1):17.

22. Rake B, Haeussler C. Did relaxing clinical trial regulation enhance the stock of scientific knowledge in India? Not necessarily. PLoS One 2019;14(1):e0210163.

23. Evangeline L, Mounica NV, Reddy VS, Ngabhushanam MV, Reddy DN, Bonthagarala B. Regulatory process and ethics for clinical trials in India (CDSCO). Pharm Innov 2017;6(4):1659.

24. Kondal A, Krishna GM, Bansal D. Clinical Trial Regulations in India: Progress and challenges arising from recent amendments to schedule Y of the Drugs and Cosmetics (D\&C) Act 1940 (D\&C Rules 1945). Pharmaceut Med 2016;30(1):1-3.

25. Imran M, Najmi AK, Rashid MF, Tabrez S, Shah MA. Clinical research regulation in India-history, development, initiatives, challenges and controversies: Still long way to go. J Pharm Bioallied Sci 2013;5(1):2-9.

26. Shivayogi P. Vulnerable population and methods for their safeguard. Perspect Clin Res 2013;4(1):53-7.

27. Mak IW, Evaniew N, Ghert M. Lost in translation: animal models and clinical trials in cancer treatment. Am J Transl Res 2014;6(2):114-8.

28. Faggion CM Jr. Animal research as a basis for clinical trials. Eur J Oral Sci 2015;123(2):61-4.

29. Curtin F, Schulz P. Assessing the benefit: risk ratio of a drugrandomized and naturalistic evidence. Dialogues Clin Neurosci 2011;13(2):183.

30. DiMasi JA, Grabowski HG, Hansen RW. Innovation in the pharmaceutical industry: new estimates of R\&D costs. J Health Econ 2016;47:20-33.

31. American Society of Clinical Oncology. American Society of Clinical Oncology policy statement: oversight of clinical research. J Clin Oncol 2003;21(12):2377-86.

32. The Solution to Drug Prices. The New York Times; 2015.

33. Bhatt A. Evolution of clinical research: a history before and beyond James Lind. Perspect Clin Res 2010;1(1):6-10.

34. Carlisle RP. Scientific American inventions and discoveriees: all the milestones in ingenuity-from the discovery of fire to the invention of the microwave oven. John Wiley and Sons, Inc; 2004.

35. Green S, Benedetti J, Smith A, Crowley J. Clinical trials in oncology. CRC press; 2012.

36. Meinert CL. ClinicalTrials: design, conduct and analysis. Oxford University Press, USA; 2012.

37. O'Rourke MF. Frederick Akbar Mahomed. Hypertension 1992;19(2):212-7.

38. Creswell JW. Educational research: Planning, conducting, and evaluating quantitative. Upper Saddle River, NJ: Prentice Hall; 2002.

39. Metcalfe NH. Sir Geoffrey Marshall (1887-1982): respiratory physician, catalyst for anaesthesia development, doctor to both Prime Minster and King, and World War I Barge Commander. J Med Biogr 2011;19(1):10-4.

40. Doll R, Hill AB. Smoking and carcinoma of the lung. Br Med J 1950;2(4682):739-48.

41. What are the different types of clinical research? Maryland: U.S. Food \& Drug Administration; 2018.

42. What is a clinical study? Bethesda, MD: National Library of Medicine, US National Institutes of Health; 2019.

43. Adams CP, Brantner VV. Estimating the cost of new drug development: is it really $\$ 802$ million? Health affairs 2006;25(2):420-8.

44. Holland J. Fixing a broken drug development process. J Commer Biotechnol 2013;19(1).

45. Phase 0 trials: a platform for drug development? Lancet 2009;374(9685):176.

46. Fisher JA. Feeding and bleeding: The institutional banalization of risk to healthy volunteers in phase I pharmaceutical clinical trials. Sci Technol Human Values 2015;40(2):199-226.

47. Norfleet E, Gad SC. Phase I clinical trials. Wiley Online Library 2010:1-10.

48. Friedman LM, Furberg C, DeMets DL, Reboussin DM, Granger CB. Fundamentals of clinical trials: Springer; 2010.

49. Porta M. A dictionary of epidemiology. Oxford university press; 2014.

50. Evans SR. Fundamentals of clinical trial design. J Exp Stroke Transl Med 2010;3(1):19.

51. Kao LS, Tyson JE, Blakely ML, Lally KP. Clinical research methodology I: introduction to randomized trials. J Am Coll Surg 2008;206(2):361-9.

52. Sessler DI, Imrey PB. Clinical research methodology 
2: observational clinical research. Anesth Analg 2015;121(4):1043-51.

53. Gandhi P. Clinical research methodology. Ind J Pharm Edu Res 2011;45(2):199-209.

54. Pannucci CJ, Wilkins EG. Identifying and avoiding bias in research. Plast Reconstr Surg 2010;126(2):619.

55. Mann CJ. Observational research methods. Research design II: cohort, cross sectional, and case-control studies. Emerg Med J 2003;20(1):54-60.

56. Schwartz S. The fallacy of the ecological fallacy: the potential misuse of a concept and the consequences. Am J Public Health 1994;84(5):819-24.

57. Khan Y. Flu, Season, Diseases Affect Trials. Appl Clin Trials 2010;19(1):40.

58. Chan AW, Tetzlaff JM, Altman DG, Laupacis A, Gotzsche PC, Krleza-Jeric K, et al. SPIRIT 2013 statement: defining standard protocol items for clinical trials. Ann Intern Med 2013;158:200-7.

59. Eysenbach G. Improving the quality of Web surveys: the Checklist for Reporting Results of Internet E-Surveys (CHERRIES). J Med Internet Res 2004;6(3):e34.

60. Fromell GJ. Good clinical practice standards: What they are and some tools to support them. Hum Gene Ther 2008;19(5):43140.

61. Faden RR, Lederer SE, Moreno JD. US Medical Researchers, the nuremberg doctors trial, and the nuremberg code: a review of findings of the advisory committee on human radiation experiments. JAMA 1996;276(20):1667-71.

62. Kim WO. Institutional review board (IRB) and ethical issues in clinical research. Korean J Anesthesiol 2012;62(1):3.

63. Lasker SP. History of clinical research and ethics. Bangladesh J Bioeth 2013;4(1):20-9.

64. Faden RR, Beauchamp TL. A history and theory of informed consent. Oxford University Press; 1986.

65. Nijhawan LP, Janodia MD, Muddukrishna BS, Bhat KM, Bairy $\mathrm{KL}$, Udupa $\mathrm{N}$, et al. Informed consent: Issues and challenges. J Adv Pharm Technol Res 2013;4(3):134.

66. Templates for informed consent forms. Geneva: World Health Organization; 2020.

67. Lipsey MW, Aiken LS. Design sensitivity: Statistical power for experimental research. Sage; 1990.

68. Dorey FJ. Statistics in brief: Statistical power: what is it and when should it be used? Clin Orthop Relat Res 2011;469(2):619-20.

69. Norwitz ER, Greenberg JA. FDA approval for use of medications in pregnancy: an uphill battle. Rev Obstet Gynecol 2011;4(2):39-41.

70. Morris ZS, Wooding S, Grant J. The answer is 17 years, what is the question: understanding time lags in translational research. J R Soc Med 2011;104(12):510-20.

71. Unger JM, Cook E, Tai E, Bleyer A. The role of clinical trial participation in cancer research: barriers, evidence, and strategies. Am Soc Clin Oncol Educ Book 2016;36:185-98.

72. Baer AR, Zon R, Devine S, Lyss AP. The clinical research team. J Oncol Pract 2011;7(3):188-92.

73. Marwah R, Van de Voorde K, Parchman J. Good clinical practice regulatory inspections: Lessons for Indian investigator sites. Perspect Clin Res 2010;1(4):151-5.

74. Borovecki A. Committees: Research ethics committees. In: Henk ten Have, editor. Encyclopedia of Global Bioethics. Switzerland: Springer; 2016.

75. Moynihan R. Who pays for the pizza? Redefining the relationships between doctors and drug companies. 2: Disentanglement. Br Med J 2003;326(7400):1193-6.

76. Davidoff F, DeAngelis CD, Drazen JM, Nicholls MG. Sponsorship, authorship and accountablility. Can Med Assoc J 2001;165(6):786-8.

77. Woolley KL, Lew RA, Stretton S, Ely JA, Bramich NJ, Keys JR, et al. Lack of involvement of medical writers and the pharmaceutical industry in publications retracted for misconduct: a systematic, controlled, retrospective study. Curr Med Res Opin 2011;27(6):1175-82.

78. Prada-Ramallal G, Takkouche B, Figueiras A. Summarising the evidence for drug safety: a methodological discussion of different meta-analysis approaches. Drug Saf 2017;40(7):54758.

79. Lang T, Siribaddana S. Clinical trials have gone global: is this a good thing?. PLoS Med. 2012;9(6):e1001228.

80. Acharya K. India: Prime Destination for Unethical Clinical Trials. Portland, USA: Inter Press Service; 2007.

81. Naik K. Clinical Trials in India: History, current regulations, and future considerations: Eastern Michigan University; 2017.

82. Annas GJ. Globalized clinical trials and informed consent. N Engl J Med 2009;360(20):2050-3.

83. Singh S, General DC. Clinical trials new horizon-India. Pharmexcil.com; 2013.

84. Sawant P, Roychowdhury V. What are the three main challenges faced by the Indian clinical market; 2010.

85. Nundy S, Gulhati CM. A new colonialism?-Conducting clinical trials in India. N Engl J Med 2005;352(16):1633-6.

86. Sharma H, Parekh S. Clinical Trials Regulations in India. Pharmaceut Reg Affairs 2012;1:e118.

87. Saxena P, Saxena R. Clinical trials: changing regulations in India. Indian J Community Med 2014;39(4):197-202.

88. Tulasi GK, Rao BS. A detailed study of patent system for protection of inventions. Indian J Pharm Sci 2008;70(5):54754.

89. Novitzke JM. The significance of clinical trials. J Vasc Interv Neurol 2008;1(1):31.

90. Ethical Guidelines for Biomedical Research on Human Participants (2006) New Delhi: Indian Council of Medical Research; 2006.

91. Ramu B, Sathish Kumar M, Ramakrishna M. Current regulatory scenario for conducting clinical trials in India. Pharmaceut Reg Affairs 2015;4(2):1-3.

92. Kumar M, Kher S. Regulatory Considerations for Conducting Clinical Trials in India. Regul Aff Focus 2007;12:26-31.

93. Gogtay NJ, Ravi R, Thatte UM. Regulatory requirements for clinical trials in India: What academicians need to know. Indian J Anaesth 2017;61(3):192-9.

94. Sanghavi D. Regulatory reforms in India. Ther Innov Regul Sci 2013;47(1):16-8.

95. Mathiharan K. Medical Research and Clinical Trials-Indian Scenario. Indian Internet J Forensic Med Toxicol 2011;9(3):6370 .

96. Laughton AH. Somewhere to run, somewhere to hide: International regulation of human subject experimentation. Duke J Comp Int Law 2007;18;181.

97. Bannenberg W. Unethical clinical trials report WEMOS \& SOMO. USA: WEMOS \& SOMO; 2006.

98. Central Ethics Committee on Human Research. Ethical guidelines for biomedical research on human subjects. New Delhi: Indian Council of Medical Research; 2000.

99. Chakravarty A, Bhavan A. Unethical clinical trials in India: 
a selective preliminary overview. Eubios J Asian Int Bioeth 2017;27:66.

100. Srinivasan S, Nikarge S. Ethical concerns in clinical trials in India: An investigation. Centre for Studies in Ethics and Rights, Mumbai; 2009.

101. Sen F, Muthuswamy V. Capacity building for clinical trials in India. Indian J Med Res 2006;124(6):605.

102. Bhan A. Clinical trial ethics in India: One step forward, two steps back. J Pharmacol Pharmacother 2012;3(2):95.

103. Military scientists tested mustard gas on Indians. UK: The Guardian newspaper; 2007.

104. Bhopal trial: Eight convicted over India gas disaster. Delhi: BBC News; 2010.

105. Rao M. Quinacrine Sterilisation Trials: A Scientific Scandal?. Econ Polit Wkly 1998:692-5.

106. Mudur G. Researchers question ethics of diabetes drug trial. $\mathrm{Br}$ Med J 2002;325(7360):353.

107. Should clinical trials be allowed in India? Business Standard, New Delhi; 2013.

108. Srinivasan S. Indian guinea pigs for sale: Outsourcing clinical trials. India Resource Center; 2004.

109. Basil B, Adetunji B, Mathews M, Budur K. Trial of risperidone in India-concerns. Br J Psychiatry 2006;188(5):489-90.

110. Shetty P. Vaccine trial's ethics criticized. Nature.com
2011;474;427-8.

111. Cressey D. India shakes up rules on clinical trials. Nature.com; 2012.

112. Urooj M, Husain GM, Khan MA, Kazmi MH. Compensation to clinical trial participants in India: A gap analysis. Int J Pharm Investig 2017;7(2):41.

113. Rajalakshmi TK. Criminal trials. Frontline 2012;29(2).

114. Indian apex court raps government over clinical trial data. Biospectrum Bureau; 2013.

115. Ghooi RB. Expert committee to formulate policy and guidelines for approval of new drugs, clinical trials and banning of drugscomments. Perspect Clin Res 2014;5(3):100-107.

116. Shankar R. Govt accepts Ranjit Roy panel report on approval of new drugs, clinical trials \& banning of drugs. Pharmabiz. com, Mumbai; 2013.

117. New Drugs and Clinical Trials Rules, 2019. Ministry of Health and Family Welfare, New Delhi, CDSCO.gov; 2019.

118. Sapra A, Lenin B, Jain K. New Drugs and Clinical Trials Rules, 2019-A Regulatory Overview. Cyril Amarchand Mangaldas Blog; 2019.

119. Shivaprakash G, Lc P. New Drugs and Clinical Trial Rules 2019, What is New? Our views from Ethical Perspective. J Assoc Physicians India 2019;67(10):75-6. 